\title{
Логические системы Лесневского
}

\author{
Н. Г. МосквицовА
}

\begin{abstract}
The paper "Lesniewski's systems of logic" is devoted to three systems of logic of the polish logician St. Lesniewski. Lesniewski intended to construct the consistent foundations of mathematics, and then his strategy was fighting with the closure of the language and ability to control the system's openness by the especial rules of the inference. In this paper we consider the systems of Protothetic, Ontology and Mereology, and also their peculiar features and Lesniewski's contribution to the logic. We briefly describe the formation of Lesniewski's systems and characteristic properties of each system.
\end{abstract}

Keywords: Lesniewski, protothetic, ontology, mereology

В конце XIX века велся поиск возможности доказательства непротиворечивости математики. Такая попытка была предпринята Г. Фреге за счет сведения математики к логике. Он опирался на теорию множеств Г. Кантора. Однако и попытка Фреге, и теория множеств Кантора оказались противоречивыми. Обнаружение противоречивости привело к поиску возможностей «спасти» от противоречий и теорию множеств, и попытку Фреге путем создания в первом случае аксиоматической теории множеств, а во втором - теории типов.

Ученик К. Твардовского и один из значительнейших представителей Львовско-Варшавской школы, Станислав Лесневский (1886-1939) проявил интерес к проблеме обоснования математики и предпринял попытку ее решения исходя из собственных философских воззрений. Но для этого необходимо было предварительно построить некий вариант точной и непротиворечивой логики. Исходя из этой задачи, им к 1925 году были построены три логические системы - Мереология, Прототетика и Онтология (исчисление имен). Исходной системой здесь является Прототетика, над которой надстраивается Онтология, а завершает всю эту иерархию Мереология. 
Своей задачей я вижу исследование сохранившихся работ самого Ст. Лесневского, его учеников и последователей, выявления характерных черт систем Лесневского и установления их научного значения.

\section{1 Становление теорий Лесневского}

Станислав Лесневский родился 30 марта 1886 года в Серпухове. Он учился в грамматической школе в Иркутске, где работал его отец, а в 1904 году вместе с еще четырьмя польскими студентами был зачислен в университет. В студенческие годы он посетил несколько лекций Г. Корнелиуса в Мюнхене.

Диссертацию Лесневский писал во Львове под руководством ведущего философа того времени К. Твардовского. Защитил диссертацию по философии в 1912 году.

Под влиянием статьи Я. Лукасевича «On the Principle of Contradiction in Aristotle», опубликованной на польском языке в Кракове в 1910 году, Лесневский заинтересовался современной формальной логикой и особенно парадоксом Рассела, которым занимался последующие одиннадцать лет.

В период с 1911 по 1914 год Лесневский опубликовал семь статей по логике на польском языке:

1. «Вклад в анализ экзистенциальных предложений», 1911;

2. «Попытка доказательства онтологического принципа противоречия», 1912;

3. «Логические исследования», 1913 (русский перевод первых вышеуказанных двух статей);

4. «Всякая ли истина вечна, или есть также истина без начала», 1913;

5. «Критика логического принципа исключенного третьего», 1913;

6. «Является ли класс классов, не подчиненный им, подчиненным самому себе», 1914;

7. «Теория множеств в «Философских основаниях» Б. Бернштейна», 1914. 
Позже Лесневский называл эти ранние работы незрелыми и неактуальными, однако уже в них прослеживается особый тип мышления, предвосхитивший идеи мереологии [10, p. VIII].

Среди интересных пунктов этих работ следует отметить:

1) новые аргументы против существования универсалий,

2) критику конвенционализма,

3) первое различение языка и метаязыка,

4) концепцию семантических категорий, позднее противопоставленную аналогичной теории Б. Рассела,

5) первый анализ представления о множестве в так называемом собирательном смысле (позднее развито внутри системы Мереологии).

В период с 1911 по 1915 год Лесневский выступил с докладами по этим семи работам перед Варшавским психологическим обществом. В одной из работ впервые представлена теория классов, позже переименованная в Мереологиию [10, Introduction, p. IX].

С 1915 по 1918 год Лесневский жил в Москве, где преподавал математику в польской средней школе (пансион мадам Якубовски). Пребывая в Москве, он прочитал много лекций на собраниях польских групп и учреждений. Среди названий этих лекций числятся - «Проблема непротиворечивой теории множеств», «Антиномии формальных теорий и языка», «Основные проблемы современной философии», «Философские основания марксизма». Именно в этот период Лесневский сформулировал и опубликовал на польском языке первый вариант формальной системы Мереологии под названием «Основания общей теории множеств», 1916.

По возвращении в Польшу Лесневский присоединяется к группе молодых математиков под руководством 3. Янушевского и С. Мазуркевича, которые занимались основаниями математики.

С 1919 по 1939 год (вплоть до самой смерти) Лесневский занимал должность профессора философии математики в Варшавском университете. 
Важный период для математической логики в Польше начался публикацией нового журнала Fundamenta Mathematicae в 1920 году. Эту дату считают датой рождения Польской школы математики. Лесневский был членом редакционной коллегии Fundamenta с самого начала до 1928 года.

С 1918 по 1927 год Лесневский читает несколько лекций, представленных в Секции логики Варшавского философского института (среди них - «Об одной из теорем теории отношений» (1918) и «О степенях грамматических функций» (1921)). Он строит первый вариант своей второй логической системы - Онтологии. Этот вариант был представлен в двух последующих работах под общим названием - «Об основаниях онтологии». Параллельно он читает две лекции в секции логики в рамках II Польского Философского Конгресса: «Об основаниях онтологии» и «Об основаниях логистики» (Львов, сентябрь 1927).

В работе «Об основаниях математики» 1927 года Лесневский анализирует концепции и замечания о множествах, высказанные Г. Кантором, Г. Фреге, Ф. Хаусдорфом, В. Серпиньским, А. Френкелем, Е. Цермело и Б. Расселом, показывая, что все они неудачны [1, с. 39]. Источник этих неудач, согласно Лесневскому, кроется в постулировании двух принципов:

1) в утверждении существования пустого множества и

2) в невразумительных попытках рассмотрения множеств как объемов понятий.

В качестве альтернативы в 1914-1916 гг. Лесневский разрабатывал Мереологию, представляющую собой теорию частей. Однако неудовлетворенность основаниями Мереологии приводит его вначале к построению более фундаментальной системы Онтологии, а затем и Прототетики, лежащей в основании обеих.

Таким образом, новая система оснований математики Лесневского состоит из трех частей: Прототетики, Онтологии и Мереологии.

Онтологию Лесневский стал разрабатывать 1919 году. Мереология, как формальная система, была построена в 1920 году, а в письменном виде предстала на суд логико-математического сообщества только в 1930-м. Онтология охватывает теорию предикатов (свойств), классов, отношений, теорию тождества. 
Построение Прототетики как самой общей из систем Лесневского, тесно связанной с Онтологией, началось в 1921 году, хотя ее идеи можно найти уже в ранних работах Лесневского. Она была аксиоматизирована в 1929 году, но к работе над ней Лесневский вернулся в 1938-м. Прототетика - это логика пропозициональных форм с кванторами, связывающими переменные любого порядка. Особенность ее состоит именно в том, что квантифицировать можно все.

У Лесневского не было необходимости вводить обычную первопорядковую логику, поскольку можно доказать, что Онтология содержит не только первопорядковую, но и 0-порядковую предикатную логику (логику высказываний) без принятия дополнительных аксиом и правил вывода [10, Introduction, p. XV].

\section{2 Прототетика}

Прототетика является обобщением пропозиционального исчисления. Простейшее обобщение состоит в добавлении к выражениям пропозиционального исчисления кванторов, связывающих пропозициональные переменные. Высказывание в Прототетике является, таким образом, замкнутой формулой. Исходные связки Прототетики - кванторы и импликация. Дальнейшее обобщение пропозиционального исчисления - это система, которую Лесневский назвал расширенной теорией дедукции.

Чтобы перейти от элементарной Прототетики к системам, теоремы которых содержат выражения, принадлежащие тем семантическим категориям, которые мы можем определить, начиная с категории предложений, мы должны добавить новые правила вывода. В качестве таких правил выступают правила верификации или экстенсиональности. В зависимости от выбора одного из этих правил мы получаем разные, но эквивалентные друг другу системы Прототетики [12, p. 49].

Согласно Лесневскому, все выражения Прототетики делятся на классы, называемые семантическими категориями. Это деление в некоторой степени связано с теорией типов: в обеих теориях деление на семантические категории позволяет избежать логических антиномий. Существенное различие между ними состоит в том, что теория типов имеет дело с индивидами, клас- 
сами и отношениями, в то время как Лесневский говорит о выражениях логики - элементах языка.

Базисная семантическая категория в Прототетике - категория высказываний. Все пропозициональные переменные принадлежат категории высказываний. Все выражения, не принадлежащие к базисной семантической категории и не являющиеся кванторами и скобками, являются функторами. Кванторы и скобки в Прототетике - синкатегорематические выражения, и им не приписывается никакая семантическая категория. Лесневский никогда не рассматривал в своих системах квантор существования. Несмотря на то что в многочисленных теоремах Онтологии Лесневского появляется квантор существования, он всегда может быть заменен последовательностью символов, состоящей из знаков отрицания и кванторов общности.

Квантификация в смысле Лесневского ближе всего к подстановочной квантификации. У Лесневского кванторы есть уже на пропозициональном уровне. Критерий Куайна («существовать - быть значением квантифицированной переменной») не применим к подстановочной квантификации, поскольку в системах Лесневского переменная пробегает по множеству, существование которого мы не можем постулировать (проблема существования универсалий). У Лесневского нет свободных переменных - все они являются разного рода сложными конструктами.

Для систем Лесневского характерно то, что определения приравниваются теоремам системы. Лесневский отказывается от расселовского понимания определения как сокращения. Определения Лесневского - это выражения, теоремы. Они записываются либо в виде эквивалентности, либо в форме двух импликаций. Введение определений автоматически делает их аксиомами системы.

Иногда введение определений изменяет саму систему таким образом, что она становится креативной. В то же время определения обладают свойством переводимости, т. е. во вновь полученной с помощью определения системе для каждой формулы, содержащей определяемый термин, всегда найдется соответствующий эквивалент формул из предыдущей системы [1, с. 36].

Стратегией Лесневского [1, с. 38] была борьба с замкнутостью 
языка. Креативность систем Лесневского, обусловленная введением и особенностью определений, влечет открытость систем. Контроль над новыми системами осуществляется посредством правил вывода (в частности, правил экстенсиональности и верификации). Эти правила позволяют сохранять открытость систем.

Все результаты, получаемые в стандартном пропозициональном исчислении, получаются и в Прототетике.

В Прототетике имеют место следующие три аксиомы:

A1. $\forall p q r((p \rightarrow q) \rightarrow((q \rightarrow r) \rightarrow(p \rightarrow r)))$

A2. $\forall p q(q \rightarrow(p \rightarrow q))$

A3. $\forall p q((p \rightarrow q) \rightarrow p) \rightarrow p))$

Все теоремы стандартного пропозиционального исчисления (с импликацией и отрицанием в качестве единственных связок) являются следствиями А1-А3 [12, р. 54].

Для формулировки некоторых характеристических теорем Прототетики требуются следующие определения:

D1. $\forall p(a s(p) \equiv p)$,

D2. $\forall p(v r(p) \equiv(p \equiv p))$,

D3. $\forall p(f l(p) \equiv(p \equiv \neg p))$,

D4. $1=\forall p(p \equiv p)$,

D5. $0=\forall p(p)$,

D1-D3 определяют функторы пропозиционального исчисления. Другие два определения определяют константы семантической категории высказываний. Символы $a s, v r, f l$ суть сокращения для функторов «утверждение» (assertio), «истина» (verum) и «ложь» (falsum) соответственно.

Приведем характеристические законы прототетики Лесневского [12, p. 55-59]:

1 Закон экстенсиональности:

(a) $\forall f, p, q((p \equiv q) \rightarrow(f(p) \equiv f(q)))$ 
Все функции от одного аргумента в элементарной Прототетике являются истинностными функциями.

2 Закон количества функиий:

(b) $\forall f((\forall p f(p) \equiv a s(p)) \vee(\forall p f(p) \equiv \operatorname{vr}(p)) \vee(\forall p f(p) \equiv$ $f l(p)) \vee(\forall p f(p) \equiv \neg p))$

Всякая пропозициональная функция от одного аргумента эквивалентна одной из четырех функций: $a s(p), \operatorname{vr}(p), f l(p)$ или $\neg p$. Этими функциями исчерпываются все альтернативы пропозициональных функций от одного аргумента.

\section{3 Закон развития:}

(c) $\forall f, p((f(1) \equiv p) \vee(f(0) \& \neg p))$

Всякая пропозициональная функция $f(p)$ после замены $p$ любым истинным высказыванием эквивалентна выражению $f(1)$, а после замены любым ложным высказыванием - $f(0)$.

4 Закон верификации:

(d) $\forall f, p((f(1) \& f(0) \rightarrow f(p))$

Всякая функция от одного аргумента, удовлетворяемая выражениями 1 и 0 , в элементарной Прототетике удовлетворяется любым выражением.

5 Закон предела функиии:

(e) $\forall f(\forall p f(p) \equiv(f(1) \& f(0))$

Эту теорему можно использовать для определения квантора общности посредством конъюнкции и терминов 1 и 0 . Термины «закон развития» и «теорема о пределе функции» заимствованы из алгебры логики [6, p. 50, 123].

6 Обобщенный закон предела функиии:

(f) $\forall f, q(\forall p f(p) \equiv(f(q) \& f(\neg q)))$. 
Пропозициональная функция от одного пропозиционального аргумента удовлетворяется любым высказыванием, если и только если она удовлетворяется двумя любыми противоречивыми высказываниями.

Законы (a)-(f) могут быть обобщены также для функций с большим числом аргументов. Все законы Прототетики являются эквивалентными друг другу.

Правила вывода в системах Прототетики, как было сказано выше, могут быть различными. Так, правило подстановки не разрешает подставлять на место сложных выражений другие выражения. Вместо свободных переменных, пробегающих по функторам любой семантической категории, подставляются константы или переменные той же семантической категории.

Правило отделения может применяться только к таким импликациям и эквивалентностям, которым не предшествуют кванторы:

Пусть высказывание $\forall p(\alpha)$ - теорема всех систем Прототетики, высказывание $\forall p(\alpha \rightarrow \beta)$ - теорема систем с единственной примитивной связкой - импликацией, а высказывание $\forall p(\alpha \equiv$ $\beta)$ - теорема системы с примитивной связкой - эквивалентностью. Поскольку правила вывода позволяют нам проносить квантор через импликацию и эквивалентность, мы получаем следующие теоремы: $\forall p(\alpha) \rightarrow \forall p(\beta)$ и $\forall p(\alpha) \equiv \forall p(\beta)$. А теперь к обоим высказываниям можно применить правило отделения.

Правило верификачии у Лесневского является обобщением правила, сформулированного Лукасевичем. Правило Лукасевича позволяет добавлять к системе пропозиционального исчисления любое высказывание, для которого выполняется следующее условие: выражения, полученные в результате подстановки в высказывание вместо одной из его пропозициональных переменных значения 1 и 0 , являются теоремами этой системы. Выражения 1 и 0 здесь выступают верификаторами пропозициональных переменных. Для обобщения правила верификации нам нужно уметь получать верификаторы для выражений любой семантической категории. Это можно сделать путем введения определения эквивалентности двух функторов, принадлежащих к любой, но общей для обоих функторов семантической категории. Схема определения выглядит так: 


$$
\forall \varphi, \psi\left((\varphi \equiv \psi) \equiv \forall \xi_{1}, \ldots, \xi_{n}\left(\varphi\left(\xi_{1} \ldots \xi_{n}\right) \equiv \psi\left(\xi_{1} \ldots \xi_{n}\right)\right)\right) .
$$

Выражения 1 и 0 суть верификаторы пропозициональной переменной, а функторы, определенные с помощью определений, имеющих вышеприведенную структуру, являются верификаторами функторной переменной $\varphi$. Эти определения содержат верификаторы аргументов функтора $\varphi$, которые имеют более низкий семантический порядок, чем переменная $\varphi$.

Правило верификации можно сформулировать так:

Пусть $\alpha-$ произвольное высказывание Прототетики. Если всякое высказывание, получаемое из а путем подстановки в $\alpha$ верификаторов вместо какого-либо из свободных вхождений функтора, будет теоремой этой системы, то а также будет теоремой системы [12, р. 84].

Первая система Прототетики $S$ содержит в качестве единственной примитивной связки импликацию, а в качестве правил вывода - правила подстановки, отделения, добавления и удаления квантора и правило верификации. Ее единственная аксиома имеет следующий вид:

$$
\text { A1. } \forall f, g(f(\forall p(p \rightarrow p)) \rightarrow(f(\forall p(p)) \rightarrow f(q))) \text {. }
$$

Другая система $S 1$ получается при замене правила верификации на правило экстенсиональности, которое гласит:

Для данного одноаргументного функтора, по крайней мере второго семантического порядка, закон экстенсиональности, сформулированный для данного функтора, является теоремой системы [12, р. 86].

Для систем $S$ и $S 1$ доказана их эквивалентность и полнота.

Помимо $S$ и $S 1$ существует система $S 2$, единственный примитивный термин которой - эквивалентность. Список ее аксиом выглядит так:
A1. $\forall p, q, r((p \equiv q) \equiv((r \equiv q) \equiv(p \equiv r)))$
A2. $\forall p, q((p \equiv q) \equiv \forall f(f(p) \equiv f(q)))$
A3. $\forall p, q((p \equiv q) \equiv(\forall f(f(p) \equiv f(q)) \equiv(p \equiv q)))$ 
A4. $\forall f(f(\forall p(p)) \equiv(f(\forall p(p) \equiv \forall p(p)) \equiv \forall q(f(\forall p(p) \equiv f(q)))))$.

Правилами вывода системы $S 2$ являются:

1. Правило отделения (если эквивалентность двух высказываний - теорема в $S 2$, и первое из этих высказываний теорема в $S 2$, то и второе высказывание - теорема $S 2$ ),

2. Правило подстановки,

3. Правило пронесения квантора общности через эквивалентности,

4. Правило эквивалентности (любой закон экстенсиональности вида эквивалентности является теоремой $S 2$ ),

5. Правило определения: любое правильно построенное определение есть теорема $S 2$; такие определения являются эквивалентностями.

Импликация в $S 2$ может быть определена в терминах эквивалентности и конъюнкции следующим образом:

$$
\forall p, q((p \rightarrow q)) \equiv((p \& q \equiv p)),
$$

где конъюнкцию, в свою очередь, можно определить двумя способами:

D1. $\forall p, q(p \& q \equiv \forall f(p \equiv(f(p) \equiv f(q)))$.

D2. $\forall p, q(p \& q \equiv \forall f(p \equiv(\forall r(p \equiv f(r)) \equiv \forall r(q \equiv f(r))))$.

Доказано, что система $S 2$ полна, а все теоремы системы $S 1$ суть теоремы $S 2$. Таким образом, три системы Прототетики полны и эквивалентны друг другу.

В результате добавления к $S 2$ правила экстенсиональности получается система $S 3$. Условная переформулировка $S 3$ была названа $S 4$ (в ней появляются новые аксиомы и меняется набор правил вывода).

Система S5 явилась окончательной формой Прототетики. В ней содержится незначительная модификация правила определения: дефиниендумы помещаются в правую сторону эквивалентности (а не слева) и используются те же правила, что и 
в S3. Различие в том, что это делает доказательства короче, поскольку позволяет применять распределение квантора общности и правило отделения к определениям, без использования теоремы о симметричности эквивалентности [15, p. 456-459].

\section{3 Онтология}

Прототетика - часть Онтологии. В обеих теориях имеется одна и та же иерархия семантических категорий.

В Онтологии соединены две теории, введенные Лесневским, теория семантических категорий и теория отношения «ह» $[7$, p. 85]. Отношение «ع» не имеет фиксированного значения. Так, в первопорядковой элементарной онтологии Лесневского отношение «в» трактуется как «есть». В других системах оно может иметь совершенно иное значение.

В работе Ст. Лесневского «Об основаниях математики» мы находим: «Я использовал термин "онтология" для разработанной мною теории, поскольку это не противоречило моей “лингвистической интуиции", именно ввиду того факта, что я сформулировал в этой теории класс "общих принципов бытия"» [13, p. 7].

В теоремах Онтологии нет пропозициональных переменных. В языке Онтологии имеются только номинальные и пропозициональные функторы, поскольку всякий функтор в системах Лесневского составляет вместе с аргументами выражение, принадлежащее либо категории высказываний, либо категории имен [13, p. 11].

Фундаментальное свойство подразделения выражений на семантические категории состоит в том, что всякая пропозиция и всякая пропозициональная функция всегда остаются пропозицией и пропозициональной функцией, если выражение, образующее их часть, заменяется выражением, принадлежащим той же семантической категории. В случае, когда заменяющее и заменяемое выражения принадлежат разным семантическим категориям, мы получаем бессмысленное выражение.

Кроме функторов пропозиционального исчисления в теоремах элементарной Онтологии появляются также константные функторы первого порядка. Так, только переменные, встречающиеся в этих теоремах, являются номинальными (индивидны- 
ми) переменными, которые могут быть ограничены кванторами. Также теоремы элементарной Онтологии содержат индивидные константы [13, p. 11].

Элементарная онтология Лесневского является полной алгеброй множеств. С формальной точки зрения, она представляет собой теорию атомарной и полной булевой алгебры [7, p. 85]. Булева алгебра атомарна, если каждый из ее элементов, отличный от 0 , содержит в себе атом (элемент, отличный от 0 и не содержащий никаких элементов, отличных от него самого и от 0-го элемента). Отдельные объекты, десигнаты непустых единичных имен, являются такими элементами. Булева алгебра полна, если для каждого класса ее элементов булева сумма этих элементов также принадлежит алгебре. В алгебре множеств такая сумма есть множество всех индивидов, каждый из которых принадлежит, по крайней мере, одному множеству данного класса. Пустые элементы булевой алгебры суть те объекты, которые могли бы быть записаны посредством пустого имени, к примеру, имени $\Lambda$, определенного таким образом:

$$
a \varepsilon \Lambda . \equiv . a \varepsilon a . \& \sim(a \varepsilon a) .
$$

Отношение $\varepsilon$ в булевой алгебре может быть определено как отношение, имеющее место между элементами А и В, если и только если A - атом, а В - элемент, содержащий А. В Онтологии Лесневского принимаются следующие два правила вывода:

1. Правило онтологического определения и

2. Правило онтологической экстенсиональности.

Первое правило - определение множества путем указания единичных множеств, которые оно содержит, т. е. определение элемента булевой алгебры посредством указания ее атомов:

$$
a \varepsilon \varphi\{\ldots\} . \equiv . a \varepsilon a \& \Psi(\ldots a \ldots) .
$$

Пропозицию «a $\varepsilon a \gg$ в булевой алгебре следует читать: « $a$ есть атом». Лесневский определяет ее как «а есть объект».

Второе правило соответствует теореме, утверждающей, что множества, состоящие из одинаковых единиц, тождественны, т. е. элементы булевой алгебры, состоящие из одинаковых атомов, тождественны: 


$$
\forall f, g((a)(a \varepsilon f\{\ldots\} \equiv a \varepsilon g\{\ldots\}) \equiv \forall \Omega(\Omega<f>\equiv \Omega<g>))[7, p .90]
$$

Правило определения обеспечивает возможность определения произвольных функций булевой алгебры. Именно Лесневский первым дал описание этих правил, и это было достижением исторической важности.

Но остаются открытыми, к примеру, философские проблемы. Лесневский строил Онтологию, рассматривая ее как формальную теорию объектов. Слово «есть», соответствующее символу «६», имеет онтологическое значение. Согласно этому значению, пропозиция формы « $a$ есть $b \gg$ истинна, если только субъект пропозиции - непустое имя, т. е. если десигнат субъекта существует в онтологическом мире. Кроме того, как мы знаем, субъект должен быть единичным именем, а предикат должен относиться к субъекту. Таким образом, следующая пропозиция истинна:

\section{Коперник есть поляк.}

Следовательно, элементарная онтология, если вслед за Лесневским считать термин «є» сокращением слова «есть», является теорией определенного реального отношения, названного отношением «быть чем-то».

Единственная аксиома Онтологии сформулирована Ст. Лесневским в 1920 г. и может быть записана следующим образом:

$\mathrm{Ax}:(x \varepsilon X) \equiv \exists y(y \varepsilon x) \& \forall y, z(y \varepsilon x \& z \varepsilon x \rightarrow y \varepsilon z) \& \forall y(y \varepsilon x \rightarrow y \varepsilon X)$.

Приведем также фундаментальный закон Онтологии:

(I) $x \varepsilon X \& y \varepsilon x \rightarrow x \varepsilon y$.

\section{4 Мереология}

Мереология - самая ранняя из систем Лесневского. Первоначально предполагалось, что она будет действовать как теория множеств. Таков был заголовок, под которым был опубликован большой ее фрагмент в Москве в 1916 г. [11].

Мереология - теория слова «часть», понимаемого как «кусок» (ріесе) некоторого объекта. Часть объекта не может быть пустой, она содержится в объекте, но не заполняет его весь. 
Хотя в этой теории используются термины «класс» и «множество», она значительно беднее других аксиоматических теорий множеств. Сам Лесневский отказался от идеи рассмотрения своей системы как теории множеств и в более поздних работах давал ей название «мереология».

С точки зрения Лесневского, существует только одно исходное отношение «быть элементом» $(x \in y)$, а отношение включения вводится исключительно по определению.

Базовое отношение «часть - целое» является составным:

$A \varepsilon \operatorname{prB}$ (читается, как «A есть часть B), где $p r$ - функтор с одним аргументом [2].

В мереологии не проходит парадокс Рассела. Поскольку у Лесневского целое рассматривается как самая большая часть, парадокс Рассела элиминируется. Рассмотрим аксиомы Мереологии.

A1. $x \varepsilon \operatorname{pr}(y) \& y \varepsilon \operatorname{pr}(z) \rightarrow x \varepsilon \operatorname{pr}(z)-$ транзитивность.

A2. $x \varepsilon \operatorname{pr}(y) \rightarrow \neg(y \varepsilon \operatorname{pr}(x))-$ антисимметричность.

A3. $x \varepsilon \operatorname{pr}(y) \rightarrow y \varepsilon y-$ непустота.

A4. $x \varepsilon K l(Y) \& z \varepsilon K l(Y) \rightarrow x=z-$ единичность класса.

Приведем также определения «быть элементом» и «быть коллективным классом», соответственно Df1 и Df2.

Df1. $x \varepsilon \operatorname{el}(y) \equiv x \varepsilon x \&(x \varepsilon \operatorname{pr}(y) \vee x=y)$,

Df2. $x \varepsilon K l(Y) \equiv x \varepsilon x \& \forall z(z \varepsilon Y \rightarrow z \varepsilon e l(x)) \& \exists z(z \varepsilon e l(x) \rightarrow$ $\exists r, t(r \varepsilon Y \& t \varepsilon \operatorname{el}(z) \& z \varepsilon e l(r)))$, где $K l(Y)$ - свойство быть коллективным классом.

Мереологию можно отнести к тем теориям, в которых имеет значение тот факт, что все элементы должны иметь эквиваленты в действительности. В подобных теориях нулевой элемент не имеет физической интерпретации. Кажется, что именно таковым было намерение Лесневского в развитии Мереологии, когда он говорил, что «часть» означает столько же, сколько «кусок», и «класс A»- столько же, сколько «груда/масса A». Таким же было намерение Х.С. Леонарда и Н. Гудмана, которые спустя 
пятнадцать лет после Лесневского и независимо от него сформулировали теорию, схожую с Мереологией, которую они назвали исчислением индивидов [9, p. 45-55]. Дж. Х. Вудгер использовал Мереологию в формализации биологии [14, p. 5-18].

Исследование трудов Лесневского представляется мне очень важным. Эта важность обусловлена тем, что логические системы Лесневского во многом предвосхитили поздние и более известные результаты современной логики. Несмотря на то что труды самого Ст. Лесневского практически не доступны и его идеи дошли до нас в интерпретации его учеников и последователей, я считаю, что историческое значение его трудов несправедливо недооценено, и необходимо восполнить этот пробел.

\section{Литература}

[1] Васюков В.Л. Формальная феноменология, М.: Наука, 1999.

[2] Васюков В.Л. Материалы лекций «Логические проблемы онтологии». Философский факультет МГУ, кафедра логики. Март 2007.

[3] Цирулис Я. П. Прототетика без типовой неопределенности выражений // Латвийский математический ежегодник. Вып. 23 (Рига). 1979. С. 166-178,

[4] Цирулис Я. П. Традиционные средства вывода в Прототетике // Латвийский математический ежегодник. Вып. 23 (Рига). 1979. С. 179-193.

[5] Chwistek L. Principle of Inconsistency in the Light of the Recent Research of Bertrand Russell // Dissertations of the Polish Academy of Science, historical and philosophical department. Vol. 30, 1912.

[6] Couturat L. L'Algebre de la logique. Paris, 1914.

[7] Grzegorczyk A. The Systems of Lesniewski in relation to contemporary logical research // Studia Logica. Vol. III, 1955.

[8] Le Blanc O.V. Lesniewski's Computative Protothetic. 1991.

[9] Leonard H. S., Goodman N. The Calculus of Individuals and its Uses //Journal of Symbolic Logic. Vol. 5, 1940.

[10] Lesniewski St. Collected Works. PWN-Polish Scientific Publishers-Warszawa. Vol. I, 1992.

[11] Lesniewski St. Podstawy ogolnej teorii mnogosci (Foundations of the General set theory), I. M., 1916.

[12] Slupecki J. St. Lesniewski's Protothetics // Studia Logica. Vol. I, Warszawa, 1953.

[13] Slupecki J. St. Lesniewski's Calculus of Names // Studia Logica. Vol.III, Warszawa, 1955.

[14] Woodger J. H. Axiomatic Method in Biology. Cambridge, 1937.

[15] Urbaniak R. Lesniewski's Systems of Logic and Mereology; History and Reevaluation. Calgary, Alberta, 2008. 